\title{
Burnout e possíveis implicações na qualidade do cuidado
}

\author{
Burnout and possible implications in the quality of care \\ Burnout y posibles implicaciones en la calidad de la atención
}

Recebido: 11/02/2021 | Revisado: 16/02/2021 | Aceito: 18/02/2021 | Publicado: 27/02/2021

Rafael da Silva Soares
ORCID: https://orcid.org/0000-0002-0796-0835
Universidade Federal Fluminense, Brasil
E-mail: rafaelsoares@id.uff.br
Jorge Luiz Lima da Silva
ORCID: https://orcid.org/0000-0002-2370-6343
Universidade Federal Fluminense, Brasil
E-mail: jorgeluiz@id.uff.br
Giulia Lemos de Almeida
ORCID: https://orcid.org/0000-0003-1783-3298
Universidade Federal Fluminense, Brasil
E-mail: giulialemos@ @id.uff.br
Natália Viana Marcondes da Silva
ORCID: https://orcid.org/0000-0002-8039-6986
Universidade Federal Fluminense, Brasil
E-mail: nataliaviana@ @id.uff.br
Marléa Chagas Moreira
ORCID: https://orcid.org/0000-0003-2706-9334
Universidade Federal do Rio de Janeiro, Brasil
E-mail: marleachagas@ gmail.com
Enéas Rangel Teixeira
ORCID: https://orcid.org/0000-0002-1721-2056
Universidade Federal Fluminense, Brasil
E-mail: eneaspsi@ hotmail.com
Karyna de Oliveira Santana Leite
ORCID: https://orcid.org/ 0000-0002-7054-4519
Universidade Salgado de Oliveira, Brasil
E-mail: karynabandeiras74@ @mail.com

\section{Resumo}

Objetivo: descrever possíveis falhas de cuidado de enfermagem relacionadas ao trabalhador acometido pela síndrome de burnout (SB). Método: estudo descritivo e exploratório que utilizou a revisão integrativa de literatura como fonte de informações, em ambiente virtual na Biblioteca Virtual de Saúde, com as bases Lilacs e Pubmed e a biblioteca virtual Scielo, em obras de diferentes nações. Resultados: a síndrome é desenvolvida em resposta a fontes crônicas de estresse emocional e interpessoal no trabalho. Caracteriza-se por cansaço emocional, despersonificação e baixa realização pessoal. Emergiram quatro categorias da análise textual: implicações e fatores relacionados à gênese; a despersonificacão e o afastamento do cuidado ao paciente; possíveis prejuízos à segurança do paciente; possível diminuição na qualidade do cuidado; e, risco à saúde mental e qualidade de vida do trabalhador. Conclusão: SB pode gerar prejuízos aos cuidados prestados aos pacientes, especialmente nos setores fechados. A despersonificação pode levar o profissional a se tornar frio, afastando-o da essência da enfermagem, o cuidado. É necessário adotar medidas preventivas e controle de doenças relacionadas ao estresse laboral.

Palavras-chave: Enfermagem; Esgotamento psicológico; Garantia da qualidade dos cuidados de saúde.

\begin{abstract}
Objective: describe possible nursing care failures related to the worker affected by the burnout syndrome (SB). Method: a descriptive and exploratory study that used the integrative literature review as a source of information in a virtual environment at the Virtual Health Library, with the Lilacs and Pubmed bases and the Scielo virtual library, in works from different nations. Results: The syndrome is developed in response to chronic sources of emotional and interpersonal stress at work. It is characterized by emotional fatigue, depersonification and low personal fulfillment. Four categories of textual analysis emerged: implications and factors related to genesis; depersonification and removal of patient care; possible damage to patient safety; possible decrease in the quality of care; and, possible risk to the worker's mental health and quality of life. Conclusion: SB can cause damage to the care provided to patients, especially in closed sectors. Depersonification can lead the professional to become cold, away from the essence of nursing, care. It is necessary to adopt preventive measures and control of diseases related to occupational stress.
\end{abstract}

Keywords: Nursing; Burnout psychological; Quality assurance health care. 


\begin{abstract}
Resumen
Objetivo: describa posibles fallas en el cuidado de enfermería relacionadas con el trabajador afectado por el síndrome de burnout (SB). Método: estudio descriptivo y exploratorio que utilizó revisión integradora de literatura como fuente de información en un entorno virtual en Biblioteca Virtual en Salud, con las bases Lilacs, Pubmed y la biblioteca virtual Scielo, en obras de diferentes naciones. Resultados: el síndrome se desarrolla en respuesta a fuentes crónicas de estrés emocional e interpersonal en el trabajo. Se caracteriza por fatiga emocional, despersificación y baja realización personal. Surgieron cuatro categorías de análisis textual: implicaciones y factores relacionados con la génesis; despersonalización y eliminación de la atención al paciente; posible daño a la seguridad del paciente; posible disminución en la calidad de la atención; y, posible riesgo para la salud mental y la calidad de vida del trabajador. Conclusión: SB puede causar daño a la atención brindada a los pacientes, especialmente en sectores cerrados. La despersonalización puede hacer que el profesional se enfríe, lejos de la esencia de la enfermería, la atención. Es necesario adoptar medidas preventivas y de control de enfermedades relacionadas con el estrés laboral.
\end{abstract}

Palabras clave: Enfermería; Agotamiento psicológico; Garantía de la calidad de atención de salud.

\title{
1. Introdução
}

O capitalismo globalizado alterou o padrão da organização do trabalho. Os países em desenvolvimento são fortemente afetados nesse sentido, porque a globalização e a implantação de novas tecnologias mantêm contratos precários, com essas nações (Pereira, 2018). Nesse cenário, as formas de adoecer e morrer dos trabalhadores estão se remodelando, o que exprime o crescimento de doenças e outras manifestações de sofrimento relacionadas ao trabalho, com repercussão na saúde mental (Lima da Silva, Acco, Almeida, Soares, Lima \& Brezolin, 2020; Trindade, Merlo, Silva, Beck \& Goes, 2017).

No Brasil, nota-se a busca por melhores remunerações e condições de trabalho, fatores que geram mudanças sociais na direção da rivalidade e individualismo exacerbado, com consequente sofrimento psíquico para os trabalhadores (Lima da Silva, Moreno, Soares, Almeida, Daher \& Teixeira, 2017; Rodrigues, Sanvador, Assis, Gomes, Bezerril \& Santos, 2017). O trabalhador deixa sua saúde em segundo plano; para que, dessa forma, consiga manter-se participante e ativo num contexto cada vez mais exigente e competitivo, mesmo com baixa remuneração. Desse modo, amplia sua carga horária, para manter o padrão de vida (Oliveira, Costa, Fernandes, Gouveia \& Rocha, 2018).

Essas demandas impulsionam os seres mergulhados na dinâmica da produtividade ao estresse constante, diminuindo os seus meios de adaptação e apoio social. Cada vez mais, o tempo livre dos profissionais é preenchido com assuntos do trabalho (Silva, Borges, Abreu, Queirós, Baptista \& Felli, 2016). No ambiente laboral do profissional de saúde também, ocorre a exigência de energia e dinamismo profissional, para que as demandas dos serviços sejam alcançadas.

O bem-estar dos trabalhadores de saúde que atuam em instituições hospitalares está propenso a diferentes estressores laborais que o afetam diretamente, como a dor, o sofrimento e, por diversas vezes, a morte (Lima da Silva, Ruback, Soares, Mota, Oliveira \& Messias, 2019). Tendo em vista todos esses possíveis cenários, cabe dizer que o profissional está exposto a cometer negligência na assistência, podendo causar danos e prejuízos, tanto para o paciente, quanto para si próprio e a instituição.

A enfermagem possui jornadas de trabalho na maioria das vezes exaustivas, devido ao grande volume de pacientes e o pouco tempo de descanso. Assim, possuem seus padrões de sono, alimentação e atividades sociais alterados, principalmente em plantões noturnos. E, muitas vezes, se dedicam a mais de um emprego, devido aos salários pouco compatíveis com a realidade social (Lima da Silva, Moreno, Soares, Almeida, Daher \& Teixeira, 2017).

A Organização Mundial da Saúde (OMS) observa que os trabalhadores da enfermagem apresentam altos níveis de ansiedade, acrescidos do risco de adoecer, provocando severos problemas de saúde mental e aumentando os casos da síndrome de burnout (SB), além de gerar transtornos de ansiedade, depressão e estresse associado (World Heatlth Organization, 2020). Além, de serem profissões alvo de inúmeros estressores psicossociais presentes no cotidiano do trabalho, como a falta de motivação, desamparo, desesperança, passividade, alienação, fadiga e estresse (Ribeiro, Oliveira, Moreira \& de Paula, 2017). 
Ademais, estudos apontam que a carga laboral excessiva é um fator de risco que estimula o desenvolvimento do estresse profissional e a Síndrome de Burnout. E, de acordo com isso, provoca o adoecimento desse funcionário no ambiente de trabalho, ocasionando como consequência direta o absenteísmo, limitando o quantitativo de trabalhadores por pacientes atendidos, refletindo em má organização do cuidado, podendo acarretar má qualidade na assistência prestada (Rodrigues, Santos \& Sousa, 2017).

A SB é definida como estresse crônico experimentado pelo indivíduo em seu contexto de trabalho, principalmente no âmbito das profissões de ensino e serviços de saúde, cuja característica essencial é o contato emocional, intenso e contínuo, com pessoas (Lima da Silva, Pereira, Santos, Bortolazzo, Rabelo \& Machado, 2018). Quando as demandas aparecem no trabalho, suas habilidades de enfrentamento e autoeficácia são reduzidas, afetando seu desempenho e criando um círculo vicioso: mais estresse; pior enfrentamento; pior desempenho e maior estresse. Se essas situações se tornarem frequentes, é muito provável que a síndrome surja (Orgambídez-Ramos, Borrego-Alés \& Ruiz-Frutos, 2018).

Como exemplo de potenciais consequências geradas pela SB, observa-se o absenteísmo o qual se tornou considerável indicador da qualidade da saúde dos trabalhadores e das condições em que o trabalho é realizado (Silva Júnior \& Merino, 2017). Em situações extremas, pode ocorrer estresse pós-traumático, abuso de álcool e drogas, e até ideias de suicídio (Moss, Good, Gozal, Kleinpell \& Sessler, 2016).

Tendo em vista a dinâmica vivenciada por esses profissionais, se desperta a necessidade de pesquisa da temática para estabelecer meios de intervenção e posterior prevenção. Nesse sentido, o estudo visa responder a seguinte questão: a literatura descreve relação entre trabalhadores com Síndrome de Burnout e a qualidade do cuidado prestado pela enfermagem? Objetivase levantar conteúdos sobre a SB entre trabalhadores de enfermagem e possíveis implicações para o cuidado.

\section{Metodologia}

Estudo de revisão integrativa de literatura baseado em obras que abordam a temática em questão, para servir de alicerce teórico para subsidiar a reflexão teórica (Souza, Silva \& Carvalho, 2010). A coleta do material para pesquisa ocorreu no primeiro semestre de 2020. A questão norteadora para este estudo foi: a literatura descreve relação entre enfermeiros com $\mathrm{SB}$ e a qualidade do cuidado prestado pela enfermagem?

O levantamento foi realizado em ambiente virtual na Biblioteca Virtual de Saúde (BVS), as bases Lilacs, Pubmed e a biblioteca virtual Scielo, utilizando-se os descritores: "enfermagem"; "garantia da qualidade dos cuidados de saúde"; "garantia da qualidade dos cuidados de saúde and (+) enfermagem"; "esgotamento psicológico". A sintaxe de pesquisa foi: "esgotamento psicológico and $(+)$ garantia da qualidade dos cuidados de saúde", "esgotamento psicológico and enfermagem", "enfermagem and $(+)$ garantia da qualidade dos cuidados de saúde".

Além da pesquisa em bases e bibliotecas virtuais em saúde, foi realizado levantamento, por meio de busca livre onde foram consultados livros, revistas impressas, entre outros, que devido à sua relevância foram incluídos nos resultados de busca com as palavras-chave descritas á cima. Os termos foram utilizados de forma conjuntas e isoladas. As obras idênticas existentes em mais de uma base foram eliminadas e, nesse caso, considerou-se seu primeiro registro. Foram considerados somente os conteúdos completos.

Na pré-seleção, foram selecionados artigos que, na leitura, demonstrassem as implicações na qualidade do cuidado devido à síndrome de burnout. As obras foram armazenadas em computador, para que em seguida fosse realizada a seleção, de acordo com leitura completa. Nessa fase, buscou-se a relação entre o conteúdo, título, resumo, e se atendiam ao objeto desta investigação.

Realizada a triagem, foram obtidos 36 artigos, publicados em português, inglês e espanhol e um manual no período de 2015 a 2020, para embasamento teórico, algumas exceções ocorreram, devido às obras clássicas conceituais. Aquelas que 
trouxeram relação com a qualidade do cuidado, ou cuidado de enfermagem foram selecionadas e organizadas no fluxograma abaixo para discussão, de acordo com análise textual nos resultados.

Figura 1. Fluxograma das obras coletadas das bases Lilacs, Pubmed, Scielo e Capes, 2020.

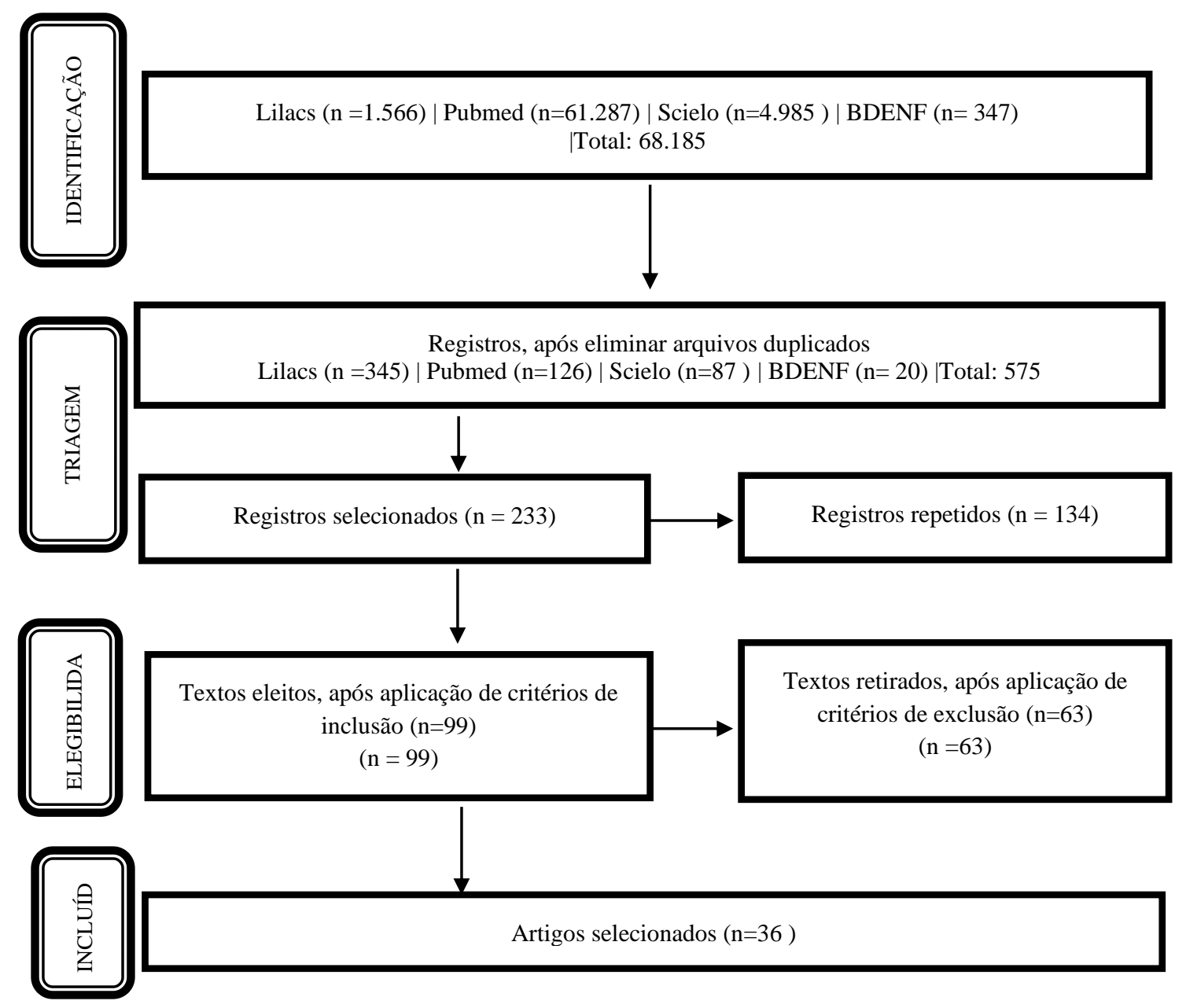

Fonte: Autores (2020).

Do total das obras, 29 compuseram a discussão dos achados, e sete delas foram utilizadas na contextualização geral deste manuscrito, desde a introdução, totalizando 36.

Os resultados foram organizados em um quadro de síntese, onde abordou-se fatores relacionados à: à gênese do burnout e implicações; despersonificação e o afastamento do cuidado ao paciente; prejuízos à segurança do paciente; diminuição na qualidade do cuidado; riscos à saúde mental e qualidade de vida do trabalhador.

\section{Resultados}

A definição e identificação do fenômeno burnout surgiu da evolução dos estudos acerca do estresse. Vários fatores podem contribuir para surgimento do esgotamento profissional, sendo sintetizados no quadro 1, de acordo com autores e suas respectivas categorias.

De acordo com a literatura, as categorias emergidas levam para origem da SB, despersonificacão e o afastamento do cuidado, possíveis prejuízos à segurança do paciente, e a diminuição na qualidade do cuidado, ricos à saúde mental e qualidade de vida do trabalhador, como pode-se observar no quadro abaixo. 
Quadro 1. Síntese das principais obras sobre aspectos relacionados à SB, capturadas nas bases virtuais Lilacs, Pubmed e Scielo, no ano de 2020.

\begin{tabular}{|c|c|}
\hline $\begin{array}{l}\text { AUTORES, ANO e } \\
\text { PAÍS }\end{array}$ & CATEGORIAS E INFORMAÇÕES RELACIONADAS \\
\hline \multicolumn{2}{|c|}{ FATORES RELACIONADOS À GÊEESE DO BURNOUT } \\
\hline $\begin{array}{l}\text { REZAEI et al, 2018- } \\
\text { Inglaterra. }\end{array}$ & Jornadas longas, baixos salários. \\
\hline $\begin{array}{l}\text { SANCHEZ et al, 2017- } \\
\text { Espanha. }\end{array}$ & Crise econômica, traumas e problemas psicológicos e mentais pregressos \\
\hline $\begin{array}{l}\text { Vasconcelos; Martino; } \\
\text { França, 2018- Brasil. }\end{array}$ & $\begin{array}{l}\text { Complexidade do trabalho, relação interpessoal, demanda elevada, } \\
\text { número reduzido de profissionais. }\end{array}$ \\
\hline $\begin{array}{l}\text { Andolhe et al, 2015- } \\
\text { Brasil. }\end{array}$ & $\begin{array}{l}\text { Descanso e sono insuficientes, condições de trabalho penoso e falta de } \\
\text { recursos. }\end{array}$ \\
\hline \multicolumn{2}{|c|}{ SOBRE A DESPERSONIFICACÃO E O AFASTAMENTO DO CUIDADO AO PACIENTE } \\
\hline Brasil, 2004. & Gestão, organização do trabalho e humanização \\
\hline $\begin{array}{l}\text { Lima da silva et al, } 2015 \text { - } \\
\text { Brasil. }\end{array}$ & \multirow{3}{*}{$\begin{array}{l}\text { Blindagem psíquica de enfrentamento, insatisfação e afastamento } \\
\text { psicológico. } \\
\text { Idem. } \\
\text { Idem. }\end{array}$} \\
\hline $\begin{array}{l}\text { Pimentel da silva et al, } \\
2015 \text {-Brasil. }\end{array}$ & \\
\hline $\begin{array}{l}\text { Loiola; Martins, } 2019 \text { - } \\
\text { Brasil. }\end{array}$ & \\
\hline $\begin{array}{l}\text { Gallegos; Toia, 2016- } \\
\text { Cuba. }\end{array}$ & Pouca realização profissional. \\
\hline \multicolumn{2}{|c|}{ SOBRE OS POSSÍVEIS PREJUÍZOS À SEGURANCCA DO PACIENTE } \\
\hline $\begin{array}{l}\text { Sousa; Mendes, } 2014 \text { - } \\
\text { Brasil. }\end{array}$ & Prezar pelo menor risco de dano possível. \\
\hline $\begin{array}{l}\text { Balsanelli; Cunha, } 2013 \text { - } \\
\text { Brasil. }\end{array}$ & Atenção a riscos de eventos adversos. \\
\hline $\begin{array}{l}\text { Rodrigues; Santos; } \\
\text { Souza, 2017- Brasil. }\end{array}$ & Fadiga e estresse crônico. \\
\hline $\begin{array}{l}\text { Novaretti et al, 2014- } \\
\text { Brasil. }\end{array}$ & Demandas urgentes e complexidade de ações. \\
\hline $\begin{array}{l}\text { Alves, Guirardello, } 2016 \\
\text {-Inglaterra. }\end{array}$ & Exaustão emocional e estabilidade no emprego. \\
\hline $\begin{array}{l}\text { Cimiotti et al, } 2012 \text { - } \\
\text { Estados Unidos. }\end{array}$ & Risco aumentado de infecções aos pacientes. \\
\hline $\begin{array}{l}\text { Galletta et al, 2016- } \\
\text { Amsterdam. }\end{array}$ & Exaustão emocional. \\
\hline Guirardello, 2017 -Brasil. & Problemas de comunicação. \\
\hline \multicolumn{2}{|c|}{ SOBRE A POSSÍVEL DIMINUICCÃO NA QUALIDADE DO CUIDADO } \\
\hline $\begin{array}{l}\text { Nogueira et al, } 2018 \text { - } \\
\text { Brasil. }\end{array}$ & Autonomia do enfermeiro. \\
\hline $\begin{array}{l}\text { Sanchez et al, 2017- } \\
\text { Espanha. }\end{array}$ & Realização profissional. \\
\hline $\begin{array}{l}\text { Liu; Aungsuroch, } 2017 \text { - } \\
\text { China. }\end{array}$ & Organização profissional e organização do trabalho. \\
\hline $\begin{array}{l}\text { Demerouti et al, 2009- } \\
\text { Holanda. }\end{array}$ & \multirow{2}{*}{$\begin{array}{l}\text { Presenteísmo e demanda elevada. } \\
\text { Idem. }\end{array}$} \\
\hline $\begin{array}{l}\text { Brborovic et al, } 2017 \text { - } \\
\text { Holanda. }\end{array}$ & \\
\hline $\begin{array}{l}\text { Chang et al, } 2017 \text { - } \\
\text { Taiwan. }\end{array}$ & Descompromisso e abandono do trabalho \\
\hline \multicolumn{2}{|c|}{ RISCOS À SAÚDE MENTAL E QUALIDADE DE VIDA DO TRABALHADOR } \\
\hline Lin et al, 2016 -Taiwan. & Depressão. \\
\hline $\begin{array}{l}\text { Vasconcelos; Martino; } \\
\text { França, } 2018 \text {-Brasil. }\end{array}$ & Danos biológico, dificuldade de concentração e atenção. \\
\hline $\begin{array}{l}\text { Andolhe et al, 2015- } \\
\text { Brasil. }\end{array}$ & Prejuízos ao sono e repouso adequados. \\
\hline
\end{tabular}




\begin{tabular}{|l|l|}
\hline $\begin{array}{l}\text { Bamoti et al, 2017 - } \\
\text { Estados Unidos. }\end{array}$ & Idem. \\
\hline $\begin{array}{l}\text { Sanchez et al, 2017 - } \\
\text { Espanha. }\end{array}$ & Esgotamento emocional. \\
\hline $\begin{array}{l}\text { Skorobogatova et al, } \\
\text { 2017- Lituânia. }\end{array}$ & Fadiga crônica. \\
\hline
\end{tabular}

Fonte: Autores (2020).

No quadro anterior, pode-se observar que quatro obras abordam o tema aspectos relacionados à origem da síndrome e implicações; cinco obras falam sobre e o afastamento do profissional do cuidado ao paciente de vido a frieza e defesas; oito obras debatem sobre os prejuízos à segurança do paciente; seis obras comentam sobre a possível diminuição na qualidade do cuidado prestado; e, seis obras apontam riscos à saúde mental e qualidade de vida do trabalhador. As informações serão discutidas na próxima seção.

\section{Discussão}

\subsection{Fatores relacionados à gênese do burnout e implicações}

Um dos principais fatores que contribuem para o aumento a prevalência de burnout entre enfermeiros é o desequilíbrio entre salário e carga de trabalho. Pesquisa realizada no Irã apontou que o desequilíbrio entre o salário e a carga de trabalho poderiam aumentar, consideravelmente, a chance de burnout (Rezaei, Karami Matin, Hajizadeh, Soroush \& Nouri, 2018).

As repercussões da crise econômica sobre a saúde da população, em geral, influenciam em aumento na maioria das doenças, especialmente problemas de saúde mental, bem como aumento do uso de drogas, trauma e suicídio. A despersonificação se correlacionou diretamente com a diminuição do salário, em estudo espanhol (Sánchez, Martínez, Lázaro Sahuquillo, Román \& Cantó, 2017). Observam-se, no cotidiano, as dificuldades encontradas pelos profissionais de saúde, de forma geral, no cenário atual no estado do Rio de Janeiro o que corrobora as constatações dos autores supracitados, o que se potencializa com a dificuldade de enfrentamento coletivo.

As relações interpessoais foram apontadas como um dos principais fatores que causam burnout. Segundo estudo realizado com enfermeiros de UTI de hospital universitário, o profissional atende a muitos pacientes de alta complexidade diariamente, resultado do insuficiente número de funcionários. O excesso de tarefas por sujeito demanda a aceleração ao realizar as atividades, diminuição do tempo de pausa e aumento na quantidade das relações interpessoais. Isso predispõe esses profissionais ao adoecimento, devido ao estresse (Vasconcelos, Martino \& França, 2018).

A insatisfação com as condições de trabalho, cansaço e sono inadequado também são fatores que podem causar burnout. Pesquisa com profissionais atuantes em UTI cirúrgica encontrou tal associação. Essas características evidenciam perfil de profissionais que podem estar mais suscetíveis ao burnout. Além disso, a capacidade para o trabalho parece prejudicada, quando os profissionais vivenciam condições de trabalho desfavoráveis (Andolhe, Barbosa, Oliveira, Costa \& Padilha, 2015).

\subsection{A Despersonificação e o afastamento do cuidado ao paciente}

A Política Nacional de Humanização (PNH) é política pública no SUS voltada para ativação de dispositivos que favoreçam ações de humanização, no âmbito da atenção e da gestão da saúde no Brasil (Ministério da Saúde, 2004). Entre as diretrizes da PNH, encontra-se a valorização do trabalho e do trabalhador. No eixo da gestão do trabalho, propõe-se a promoção de ações que assegurem a participação dos trabalhadores nos processos de discussão e decisão, reconhecendo, 
fortalecendo e valorizando seu compromisso com o processo de produção de saúde, e seu crescimento profissional. A gestão coletiva das situações de trabalho é critério fundamental para a promoção de saúde, e a prevenção de adoecimento (Ministério da Saúde, 2004).

A despersonificação constitui estratégia de enfrentamento que surge, posteriormente aos sentimentos de esgotamento emocional e baixa realização pessoal. Utilizando-se mecanismos, o trabalhador pode se distanciar de si pscicologicamente, tornando-se frio e cínico, de modo a tratar os clientes e colegas como objetos e merecedores dos problemas que possuem. Por fim, ocorre o afastamento psicológico, como blindagem psíquica de enfrentamento, desenvolvida para lidar com o esgotamento emocional (Lima da Silva, Soares, Costa, Ramos, Lima \& Teixeira, 2015; Pimentel da Silva, Barbosa, Souza da Silva \& Patrício, 2015; Loiola \& Martins, 2019).

Pesquisa peruana correlacionou negativamente a despersonificação com baixa realização profissional, ou seja, quanto maior a despersonificação, menor realização profissional. Nesse sentido, a falta de contato dos enfermeiros com a realidade, constitui característica da despersonificação, manifestada pela falta de interesse pelo paciente, e consequente afastamento do trabalho que é realizado (Gallegos \& Toia, 2016). Com isso, esse afastamento, mostra-se como "descuidado" aos pacientes; e, consequentemente, pode afetar a realização trabalhadores, em suas atividades diárias de cuidado às pessoas. Nesse aspecto, a síndrome de burnout influencia negativamente dois aspectos da PNH: a assistência e a valorização profissional. Ou seja, a despersonificação afasta o profissional de enfermagem do cuidado e a baixa realização profissional atrapalha a articulação entre os trabalhadores, impedindo seu crescimento e satisfação profissional.

\subsection{Possíveis prejuízos à segurança do paciente}

A segurança do paciente versa sobre a melhoria da qualidade nos serviços de saúde e na disseminação das boas práticas na assistência (Rodrigues, Santos \& Sousa, 2017). A preocupação com a segurança do paciente, compreendida como a redução a um mínimo aceitável do risco de dano desnecessário associado ao cuidado, sendo atributo da qualidade do cuidado. A segurança é indispensável para o cuidado de qualidade (Sousa \& Mendes, 2014).

As UTI, por exemplo, são setores específicos que atendem doentes em estado grave de saúde e exigem da equipe interdisciplinar muitas competências que, para serem exercidas, necessitam de condições adequadas (Balsanelli \& Cunha, 2013). Eventos adversos podem acontecer em qualquer setor da assistência à saúde, mas pacientes em UTI são particularmente vulneráveis a essas complicações, em função da própria gravidade, necessidade de decisões de alto risco, utilização de arsenal medicamentoso mais complexo, e número elevado de procedimentos diagnósticos e intervenções realizadas (Novaretti, Santos, Quitério \& Daud-Gallotti, 2014).

$\mathrm{Na}$ análise dos fatores relacionados aos eventos adversos em pacientes em UTI, destaca-se o papel da enfermagem, uma vez que este é o local em que há maior demanda para as atividades, exigência de ações rápidas e observação continuada por parte desses profissionais. Logo, a sobrecarga de demandas e a ocorrência de SB podem prejudicar a percepção de riscos, o que pode afetar segurança do paciente (Novaretti, Santos, Quitério \& Daud-Gallotti, 2014).

Desse modo, a fadiga e o estresse desses profissionais podem interagir para produzir qualidade mais baixa do atendimento ao paciente e, em consequência disso, ocorrer o aumento da frequência de incidentes clínicos (Alves \& Guirardello, 2016).

Quanto à relação entre o burnout e a segurança do paciente, pesquisa indica que quanto menor o nível de exaustão emocional, melhor a percepção do clima de segurança e maior satisfação no trabalho. Além disso, quanto mais satisfeitos os profissionais estão com o trabalho, mais positivo é o clima de segurança) (Alves \& Guirardello, 2016).

Pesquisa desenvolvida nos Estados Unidos encontrou associação entre enfermeiros com burnout e infecções em sítio cirúrgico e do trato urinário de pacientes. Logo, reduzir o burnout entre enfermeiros é estratégia promissora para ajudar a 
controlar infecções em UTI (Cimiotti, Aiken, Sloane \& Wu, 2012). Ambientes favoráveis à prática profissional da equipe de enfermagem podem resultar em menores níveis de exaustão emocional, melhor qualidade do cuidado e percepção positiva sobre atitudes de segurança (Guirardello, 2017).

O burnout pode, indiretamente, pela mediação da qualidade da comunicação em equipe, afetar infecções associadas aos cuidados de saúde nas UTI, como resultado da qualidade das condições de trabalho, segundo pesquisa italiana (Galletta et al, 2016).

Ou seja, fatores como insatisfação, comunicação ineficaz, carga de trabalho excessiva e ambiente laboral com condições precárias contribuem para o estresse e SB, refletindo no cotidiano desses trabalhadores, tornando-os mais vulneráveis a desenvolver assistência insegura. Dessa forma, é necessário implementar estratégias de gestão voltadas para a segurança do paciente, juntamente com a promoção do bem-estar do trabalhador, ambiente e condições laborais adequadas, a fim de aprimorar o padrão de qualidade dos cuidados prestados aos pacientes.

\subsection{Possível diminuição na qualidade do cuidado}

Naturalmente, o trabalhador de enfermagem afetado pela síndrome de burnout não produzirá cuidado de qualidade, haja vista sua exaustão emocional, que aumenta o desgaste físico e emocional; a diminuição da realização profissional, perdendo o sentimento de recompensa por seu cuidado prestado; e a despersonificação, que pode levar o profissional a ficar emocionalmente mais rígido e tratar pessoas como objetos, afastando-o do cuidado.

Pesquisa identificou que existe relação positiva e direta entre autonomia e burnout. Quanto maior o senso de autonomia, maior a percepção de realização pessoal, uma vez que o maior senso de autonomia pode ter efeito protetor no desenvolvimento do burnout, logo essa percepção tem efeito protetor para a baixa realização pessoal (Nogueira, Cardoso de Sousa, de Souza Guedes, Turrini \& dos Santos, 2018). A liberdade do enfermeiro para resolver problemas, que afetam a qualidade da assistência de enfermagem, acaba por favorecer ao trabalhador e a qualidade do cuidado.

A relação entre as dimensões de burnout e as variáveis de trabalho mostra que o esgotamento emocional é maior entre aqueles que percebem o ambiente de trabalho como ruim/muito ruim, em comparação com aqueles que o consideram regular, bom/muito bom. O mesmo acontece com a despersonificação. Em termos de realização pessoal, essa é maior naqueles que consideram seu ambiente de trabalho como bom/muito bom, em comparação com aqueles que o percebem como ruim/muito ruim, segundo estudo espanhol (Vasconcelos, Martino \& França, 2018).

Estudo chinês identificou que o ambiente de trabalho influencia a satisfação profissional, que a satisfação profissional influencia o burnout, e que o burnout afeta negativamente a percepção das enfermeiras sobre o grau de excelência nos serviços de enfermagem (Liu \& Aungsuroch, 2017). Ou seja, tanto a percepção do ambiente de trabalho como ruim, como a insatisfação laboral, associam-se ao burnout. Isso quer dizer que a síndrome influencia diretamente, na diminuição da qualidade do cuidado.

Pesquisa na Holanda demonstrou que as demandas de trabalho e o burnout exibiram relação com presenteísmo entre enfermeiros. As demandas elevadas de trabalho causaram mais presenteísmo, assim como despersonificação, em longo do prazo (Demerouti, Le Blanc, Bakker, Schaufeli \& Hox, 2009; Brborović, Daka, Dakaj \& Brborović, 2017). Desse modo, o excesso de trabalho favorece o presenteísmo; que, por sua vez, limita a produtividade tanto em quantidade, quanto na qualidade do trabalho. Diminui o rendimento físico e mental do trabalhador, aumenta o sentimento de desgaste e frieza, bem como a possibilidade de causar prejuízos à atenção no desenvolvimento da assistência.

Estudo desenvolvido em Taiwan identificou que burnout diminui o compromisso profissional. O compromisso profissional foi definido como o grau em que um indivíduo é dedicado e orgulhoso de ser membro de uma profissão, e como acredita nos valores e objetivos da profissão (Chang, Shyu, Wong, Chu, Lo \& Teng, 2017). Isso significa que os profissionais 
de enfermagem com burnout desejam abandonar a instituição onde trabalham, mas não gostariam de desistir da enfermagem. Ou seja, buscam melhores condições de trabalho, mas, em outras organizações.

Em consonância, pesquisa chinesa revelou que profissionais com burnout têm mais intenção de abandonar o trabalho (Liu \& Aungsuroch, 2017). Com isso, forma-se círculo vicioso, no qual o trabalhador insatisfeito abandona o trabalho, deixando seus colegas em defasagem, até a chegada de novos profissionais naquele ambiente, para que se submetam as mesmas condições difíceis de trabalho.

\subsection{Riscos à saúde mental e qualidade de vida do trabalhador}

A depressão influi no desempenho profissional do das enfermeiras. Pesquisa em Taiwan revelou que os níveis de burnout e depressão ocupacional também foram correlacionados positivamente. Em nível organizacional, a depressão aumenta as taxas de rotatividade de enfermagem; e, indiretamente, aumenta a carga de trabalho de outras enfermeiras. Ao longo do tempo, é formado ciclo periódico de baixa satisfação no trabalho, alta rotatividade e recursos humanos insuficientes (Lin, Lin, Cheng, Wu \& Ou-Yang, 2016).

Foi verificada a correlação entre os escores das dimensões do burnout e o escore total de sintomatologia depressiva, pode-se dizer que existiu correlação, em que, quanto maior foi o nível de exaustão emocional e de despersonificação, maior a sintomatologia depressiva; e, quanto menor a realização profissional (inversamente proporcional), maior a sintomatologia depressiva (Vasconcelos, Martino \& França, 2018).

Pesquisas no Brasil, Estados Unidos e Espanha revelaram impacto do burnout na qualidade do sono (Sánchez, Martínez, Lázaro Sahuquillo, Román \& Cantó, 2017; Andolhe, Barbosa, Oliveira, Costa \& Padilha, 2015; Bamonti et al, 2017). A privação do sono provoca diversos distúrbios, sendo os principais: gastrointestinais; cardiovasculares; cognitivos; flutuações de humor; comprometimento do desempenho das atividades; pessoais; sociais e do trabalho, pois diminui a capacidade de concentração e atenção. Assim, as horas de sono adequadas para a restauração do organismo desgastado pela jornada de trabalho parecem contribuir para evitar os sinais e sintomas de desgaste físico, sobretudo, do estresse e burnout, citados anteriormente, e são de extrema importância para a saúde do indivíduo (Andolhe, Barbosa, Oliveira, Costa \& Padilha, 2015).

Quanto às dimensões do burnout, estudo espanhol evidenciou que os profissionais que experimentaram mudança nas condições de trabalho têm maior esgotamento emocional. Ocorre do mesmo modo naqueles que tiveram a morte de parente próximo, mudanças na situação econômica, nas atividades sociais e nos hábitos alimentares (Andolhe, Barbosa, Oliveira, Costa \& Padilha, 2015).

Em pesquisa realizada na Lituânia, a insônia, falta de descanso, nervosismo e cansaço foram as queixas mais comuns dos profissionais. A análise revelou que apenas o cansaço pode ser considerado sintoma associado ao burnout, todos os outros sintomas não tiveram associação estatística (Skorobogatova, Žemaitienè, Šmigelskas \& Tamelienè, 2017).

A situação econômica afetou os profissionais de enfermagem na Espanha, de modo que, a partir de 2010, muitas administrações aproveitam a situação para reduzir os recursos humanos e materiais como medida imediata de poupança, gerando sobrecarga de trabalho e piora dos cuidados prestados. Essa medida, adicionada à redução ou congelamento de salários, aumento da carga horária, diminuição de pessoal, deteriorou o ambiente de trabalho e provocou desmotivação em nível profissional. O esgotamento emocional foi a dimensão mais influente no aparecimento da SB (Sánchez, Martínez, Lázaro Sahuquillo, Román \& Cantó, 2017).

\section{Conclusão}

De acordo com a literatura, o burnout pode gerar prejuízos aos cuidados prestados aos pacientes, especialmente nos 
setores fechados. A despersonificação pode levar o profissional a se tornar frio, afastando-o do cuidado. Além disso, o esgotamento físico e emocional dos trabalhadores pode induzi-los ao erro, negligência, falta de zelo, deste modo, repercutindo negativamente na qualidade da assistência prestada e à segurança do paciente.

Os prejuízos do burnout extrapolam a esfera profissional, podendo causar prejuízos na qualidade de vida do trabalhador. Deste modo, são imperativos esforços em suprir as necessidades desses profissionais. Há necessidade de execução das várias políticas públicas com mais eficácia e responsabilidade por parte das autoridades de saúde e dos empregadores. É necessário adotar medidas preventivas e de controle de doenças relacionadas ao estresse laboral.

Este trabalho pretendeu contribuir para compreensão das consequências do estresse emocional e interpessoal, na prática do cuidado. Pode-se observar, por meio da pesquisa bibliográfica, como a saúde mental influencia na assistência e cuidado ao paciente. Portanto, é importante que mais estudos sobre o assunto para que se amplie a reflexão sobre a qualidade do atendimento.

Por fim, a partir dos resultados deste estudo, novas pesquisas podem ser propostas para estudar a síndrome de burnout, de modo a se atentar para fatores que possam evitar o aumento da prevalência de doenças relacionadas ao estresse no trabalho.

\section{Referências}

Alves, D. F. S., \& Guirardello, E. B. (2016). Safety climate, emotional exhaustion and job satisfaction among Brazilian paediatric professional nurses. Int Nurs Rev, 63 (3), 328-335.

Andolhe, R., Barbosa, R. L., Oliveira, E. M. D., Costa, A. L. S., \& Padilha, K. G. (2015). Estresse, coping e burnout da equipe de enfermagem de unidades de terapia intensiva: fatores associados. Rev Esc Enferm USP, 49 (spe), 58-64.

Balsanelli, A, P., \& Cunha, I. C. K. O. (2013). O ambiente de trabalho em unidades de terapia intensiva privadas e públicas. Acta Paul Enferm, 26 (6), 561568.

Bamonti, P., Conti, E., Cavanagh, C., Gerolimatos, L., Gregg, J., Goulet, C., Pifer, M., \& Edelstein, B. (2017). Coping, cognitive emotion regulation, and burnout in long-term care nursing staff: a preliminary study. J Appl Gerontol, 38 (1), 92-111.

Brborović, H., Daka, Q., Dakaj, K., \& Brborović, O. (2017). Antecedents and associations of sickness presenteeism and sickness absenteeism in nurses: a systematic review. Int J Nurs Pract, 23 (6), e12598.

Chang, H. Y., Shyu, Y. I. L., Wong, M. K., Chu, T. L., Lo, Y. Y., \& Teng, C. I. (2017). How does burnout impact the three components of nursing professional commitment? Scand J Caring Sci, 31 (4), 1003-1011

Cimiotti, J. P., Aiken, L. H., Sloane, D. M., \& Wu, E. S. (2012). Nurse staffing, burnout, and health care-associated infection. Am J Infect Control, 40 (6), 486-490.

Demerouti, E., Le Blanc, P. M., Bakker, A. B., Schaufeli, W. B., \& Hox, J. (2009). Present but sick: A three-wave study on job demands, presenteeism and burnout. Career Dev Int, 14 (1), 50-68.

Demerouti, E., Le Blanc, P. M., Bakker, A. B., Schaufeli, W. B., \& Hox, J. (2009). Present but sick: A three-wave study on job demands, presenteeism and burnout. Career Dev Int, 14 (1), 50-68.

Gallegos, W. L. A., \& Toia, A. M. C. (2016). Síndrome de burnout en personal de enfermería de Arequipa. Rev. Cubana Salud Publ, 42 (4), 559-74.

Galletta, M., Portoghese, I., D’Aloja, E., Mereu, A., Contu, P., Coppola, R. C., Finco, G., \& Campagna, M. (2016). Relationship between job burnout, psychosocial factors and health care-associated infections in critical care units. Intensive Crit Care Nurs, 34, 59-66.

Guirardello, E. B. (2017). Impacto do ambiente de cuidados críticos no burnout, percepção da qualidade do cuidado e atitude de segurança da equipe de enfermagem. Rev Latinoam Enferm, 25, 1-7.

Lima da Silva, J. L., Ruback, S. P., Soares, R. S., Mota, C. P., Oliveira, M. A., \& Messias, C. M. (2019). Burnout entre profissionais de enfermagem em setores fechados. Saúde Coletiva. (48), 1375-1381.

Lima da Silva, J. L., Pereira, L. C., Santos, M. P., Bortolazzo, P. A. A. B., Rabelo, T. S., \& Machado, E. A. (2018). Prevalência da síndrome de Burnout entre professores da Escola Estadual em Niterói, Brasil. Enfermería actual de Costa Rica, (34), 14-25.

Lima da Silva, J. L., Acco, A. H., Almeida, G. L., Soares, R. F., Lima, V. F., \& Brezolin, C. A. (2020). Aspectos psicossociais e relação com sedentarismo entre trabalhadores hidroviários. Braz. J. of Develop, 6 (6), 33252-33263.

Lima da Silva, J. L., Moreno, R. F., Soares, R. S., Almeida, J. A., Daher, D. V., \& Teixeira, E. R. (2017). Prevalência de transtornos mentais comuns entre trabalhadores marítimos do Rio de Janeiro. J. Res.: Fundam. Care. Online, 9 (3), 676-681. 
Lima da Silva, J. L., Soares, R. S., Costa, F. S., Ramos, D. S., Lima, F. B., \& Teixeira, L. R. (2015). Fatores psicossociais e prevalência da síndrome de burnout entre trabalhadores de enfermagem intensivistas. Rev. bras. ter. intensiva, 27 (2), 125-133.

Lin, T. C., Lin, H. S., Cheng, S. F., Wu, L. M., \& Ou-Yang, M. C. (2016). Work stress, occupational burnout and depression levels: a clinical study of paediatric intensive care unit nurses in Taiwan. J Clin Nurs, 25 (7-8), 1120-30.

Liu, Y., \& Aungsuroch, Y. (2017). Factors influencing nurse-assessed quality nursing care: a cross-sectional study in hospitals. J Adv Nurs, 74 (4), $935-945$.

Loiola, E., \& Martins, M. D. C. (2019). Autoeficácia no trabalho e síndrome de burnout em profissionais de enfermagem. Psicologia, Saúde \& Doenças, 20 (3): 813-823.

Moss, M., Good, V. S., Gozal, D., Kleinpell, R., \& Sessler, C. N. (2016). An official critical care societies collaborative statement: burnout syndrome in critical care health care professionals: a call for action. Crit Care Med, 25 (4), 368-76.

Ministério da Saúde. (2004). Humaniza SUS: Política Nacional de Humanização: a humanização como eixo norteador das práticas de atenção e gestão em todas as instâncias do SUS. Brasília: Ministério da Saúde.

Novaretti, M. C. Z., Santos, E. D. V., Quitério, L. M., \& Daud-Gallotti, R. M. (2014). Sobrecarga de trabalho da Enfermagem e incidentes e eventos adversos em pacientes internados em UTI. Rev Bras Enferm, 67 (5), 692-9.

Nogueira, L. S., Cardoso de Sousa, R. M., de Souza Guedes, E., Turrini, R. N. T., \& dos Santos, M. A. (2018). Burnout e ambiente de trabalho de enfermeiros em instituições públicas de saúde. Rev Bras Enferm, 71 (2), 358-65.

Orgambídez-Ramos, A., Borrego-Alés, Y., \& Ruiz-Frutos, C. (2018). Empoderamento, vulnerabilidade ao estresse e burnout em enfermeiros portugueses. Cienc e Saúde Colet, 23 (1), 259-66.

Oliveira, A. L. C. B., Costa, G. R., Fernandes, M. A., Gouveia, M. T. O., \& Rocha, S. S. (2018). Presenteísmo, fatores de risco e repercussões na saúde do trabalhador de enfermagem. Av Enferm., 36 (1), 79-87.

Pimentel da Silva, R., Barbosa, S. C., Souza da Silva, S., \& Patrício, D. F. (2015). Burnout e estratégias de enfrentamento em profissionais de enfermagem. Arq. bras. psicol., 67(1):130-145.

Pereira, L. C. B. (2018). Globalização e competição: por que alguns países emergentes têm sucesso e outros não. Alta Books.

Rezaei, S., Karami Matin, B., Hajizadeh, M., Soroush, A., \& Nouri, B. (2018). Prevalence of burnout among nurses in Iran: a systematic review and metaanalysis. Int Nurs Rev, 65 (3), 361-369.

Ribeiro, L. D. C. C., Oliveira, T. C., Moreira, S. A., \& de Paula, F. A. (2017). Construção e validação de manual sobre Burnout em professores. Revista de Enfermagem do Centro-Oeste Mineiro, 7, e1317.

Rodrigues, C. C. F. M., Sanvador, P. T. C. O., Assis, Y. M. S., Gomes, A. T. L., Bezerril, M. S., \& Santos, V. E. P. (2017). Estresse entre os membros da equipe de enfermagem. Rev Enferm UFPE, 11 (2), 601-8.

Rodrigues, C. C. F. M., Santos, V. E. P., \& Sousa, P. (2017). Segurança do paciente e enfermagem: interface com estresse e Síndrome de Burnout. Rev. Bras. Enferm., 70 (5), 1083-1088.

Rodrigues, C. C. F. M., Santos, V. E. P., \& Sousa, P. (2017). Segurança do paciente e enfermagem: interface com estresse e Síndrome de Burnout. Rev Bras Enferm, 70 (5), 1141-7.

Sánchez, J. M., Martínez, N. D. A., Lázaro Sahuquillo, M., Román, A. C., \& Cantó, M. M. (2017). Análisis de impacto de la crisis económica sobre el síndrome de Burnout y resiliencia en el personal de enfermería. Enferm Glob, 16 (2), 315-35.

Silva Júnior, F. F., \& Merino, E. A. D. (2017). Proposta de gestão do absenteísmo da enfermagem hospitalar: revisão sistemática. Acta Paul Enferm, 30 (5), 546-53.

Sousa, P., \& Mendes, W. (2014). Segurança do paciente: conhecendo os riscos nas organizações de saúde. Rio de Janeiro: EAD/ENSP.

Silva, S. M., Borges, E., Abreu, M., Queirós, C., Baptista, P., \& Felli, V. (2016). Relação entre resiliência e burnout: Promoção da saúde mental e ocupacional dos enfermeiros. Revista Portuguesa de Enfermagem de Saúde Mental, (16), 41-48.

Souza, M. T., Silva, M. D., \& Carvalho, R. (2010). Revisão integrativa: o que é e como fazer. einstein., 8 (1), 102-6.

Skorobogatova, N., Žemaitienė, N., Šmigelskas, K., \& Tameliené, R. (2017). Professional burnout and concurrent health complaints in neonatal nursing. Open Med, 12, 328-334.

Trindade, L. R., Merlo, A. R. C., Silva, R. M., Beck, C. L. C., \& Goes, N. C. (2017). Fatores de adoecimento dos trabalhadores da saúde: revisão integrativa. Cien Cuid Saúde, 16 (4).

Vasconcelos, E. M., Martino, M. M. F., \& França, S. P. S. (2018). Burnout e sintomatologia depressiva em enfermeiros de terapia intensiva: análise de relação. Rev Bras Enferm, 71 (1), 147-5.

World Heatlth Organization. (2020). Actualización de la estrategia frente a la COVID-19. WHO. 